\title{
ON THE EXISTENCE OF PERIODIC SOLUTIONS FOR NONCONVEX-VALUED DIFFERENTIAL INCLUSIONS IN $\mathbb{R}^{N}$
}

\author{
SHOUCHUAN HU AND NIKOLAOS S. PAPAGEORGIOU
}

(Communicated by Hal L. Smith)

\begin{abstract}
In this paper we investigate the existence of periodic solutions for differential inclusions with nonconvex-valued orientor field. Using a tangential condition and directionally continuous selectors, we establish the existence of periodic trajectories.
\end{abstract}

\section{INTRODUCTION}

In this paper, we investigate the following multivalued periodic problem defined on $[0, b] \times \mathbb{R}^{N}$ :

$$
\left\{\begin{array}{c}
\dot{x}(t) \in F(t, x(t)) \text { a.e. } \\
x(0)=x(b) .
\end{array}\right\}
$$

Here $F: T \times \mathbb{R}^{N} \rightarrow 2^{\mathbb{R}^{N}} \backslash\{\varnothing\}$ is a set-valued vector field (orientor field), which has closed but not necessarily convex values. All earlier results on (1) assumed that the orientor field is convex-valued. We refer to the works of Haddad-Lasry [6] (Theorem B-II-1), Aubin-Cellina [1] (Theorem 4, p. 237), Macki-Nistri-Zecca [10] and Plaskacz [14] (Theorem 4.5).

In this paper using directionally continuous selectors for the orientor field $F(t, x)$ (cf. Bressan [3]) and a Nagumo type tangential condition, analogous to the one employed in [1], [6] and [14], we are able to establish the existence of solutions for (1). The approach of Macki-Nistri-Zecca [10] was based on degree-theoretic techniques.

\section{Preliminaries}

In what follows by $P_{k(c)}\left(\mathbb{R}^{N}\right)$ we will denote the collection of all nonempty, compact (and convex) subsets of $\mathbb{R}^{N}$. A multifunction (set-valued function)

Received by the editors March 16, 1994.

1991 Mathematics Subject Classification. Primary 34C25, 34A60.

Key words and phrases. Scorza-Dragoni property, directionally continuous selector, lower semicontinuous multifunction, periodic solution, tangent cone, tangential condition.

The work of the second author was done while he was visiting the University of Florence, Italy. Financial support was provided by CNR. The author wishes to thank Professor F. S. DeBlasi and Professor G. Pianigiani for their warm hospitality and for their help during the preparation of this work. 
$F: T \times \mathbb{R}^{N} \rightarrow P_{k}\left(\mathbb{R}^{N}\right)$ is said to be measurable, if for all $z \in \mathbb{R}^{N},(t, x) \rightarrow$ $d(z, F(t, x))=\inf \{\|z-v\|: v \in F(t, x)\}$ is measurable.

Let $V, W$ be Hausdorff topological spaces and $G: Y \rightarrow 2^{Z} \backslash\{\varnothing\}$. We say that $G(\cdot)$ is lower semicontinuous (l.s.c.) (resp. upper semicontinuous (u.s.c.)), if for all $U \subseteq W$ open the set $G^{-}(U)=\{v \in V: G(v) \cap U \neq \varnothing\}$ (resp. $\left.G^{+}(U)=\{v \in V: G(v) \subseteq U\}\right)$ is open in $V$. We will say that $G(\cdot)$ has an open graph, if the set $\operatorname{Gr} G=\{(v, w) \in V \times W: w \in G(v)\}$ is open in $V \times W$ furnished with the product topology.

Let $K$ be a nonempty and closed subset of $\mathbb{R}^{N}$ and let $x \in K$. The Bouligand tangent cone to $K$ at $x, T_{K}(x)$, is defined by

$$
T_{K}(x)=\left\{v \in \mathbb{R}^{N}: \liminf _{\lambda \downarrow 0} \frac{d(x+\lambda v, K)}{\lambda}=0\right\} .
$$

It is immediate from this definition that $T_{K}(x)$ is a closed, but not necessarily convex, cone in $\mathbb{R}^{N}$. It is convex if $K$ is convex or more generally locally convex at $x$.

Now let $T=[0, b]$ and let $K: T \rightarrow P_{k}\left(\mathbb{R}^{N}\right)$. Let $(t, x) \in \operatorname{Gr} K=\{(s, y) \in$ $\left.T \times \mathbb{R}^{N}: y \in K(s)\right\}$ (the graph of $\left.K\right)$. The "contingent derivative" of $K(\cdot)$ at $(t, x), D K(t, x)$, is the set-valued map whose graph is the Bouligand tangent cone to the graph of $K(\cdot)$ at $(t, x)$; i.e. $v \in D K(t, x)(\tau)$ if and only if $(\tau, v) \in$ $T_{\mathrm{Gr} K}(t, x)$ (see Aubin-Cellina [1], Chapter 4).

Finally recall that on $P_{k}\left(\mathbb{R}^{N}\right)$ we can define a metric, known in the literature as the Hausdorff metric, by setting

$$
h(A, B)=\max \left[\sup _{a \in A} d(a, B), \sup _{b \in B} d(b, A)\right] .
$$

Recall that $\left(P_{k}\left(\mathbb{R}^{N}\right), h\right)$ is a Polish space (i.e. a complete, separable metric space) and $P_{k c}\left(\mathbb{R}^{N}\right)$ is a closed subset of it. We will say that a multifunction $K$ : $T \rightarrow P_{k}\left(\mathbb{R}^{N}\right)$ is Hausdorff-Lipschitz ( $h$-Lipschitz), if $h\left(K\left(t^{\prime}\right), K(t)\right) \leq k\left|t^{\prime}-t\right|$ for some $k>0$ and all $t, t^{\prime} \in T$.

\section{Auxiliary RESUlts}

In this section we prove some auxiliary results that we will need in the proof of our main theorem (Section 4).

We start with a Scorza-Dragoni type property for measurable orientor fields $F(t, x)$, which are 1.s.c. in $x$.

Proposition 3.1. If $T=[0, b], X$ is a metric space, $Z$ is a Polish space and $F: T \times X \rightarrow 2^{Z} \backslash\{\varnothing\}$ is a multifunction with closed values such that

(1) $(t, x) \rightarrow F(t, x)$ is measurable,

(2) $x \rightarrow F(t, x)$ is l.s.c.,

then for every $\varepsilon>0$, there exists $T_{\varepsilon} \subseteq T$ compact with $\lambda\left(T_{\varepsilon}^{c}\right)<\varepsilon$ such that $\left.F\right|_{T_{\varepsilon} \times X}$ is l.s.c. (here $\lambda(\cdot)$ denotes the Lebesgue measure on $\left.T\right)$.

Proof. Consider the function $\gamma: T \times X \times Z \rightarrow \mathbb{R}_{+}$defined by $\gamma(t, x, z)=$ $d(z, F(t, x))$. Because of the measurability hypothesis on $F(t, x),(t, x) \rightarrow$ $\gamma(t, x, z)$ is measurable, while clearly $z \rightarrow \gamma(t, x, z)$ is continuous. So $(t, x, z) \rightarrow \gamma(t, x, z)$ is measurable. Furthermore, if $\left(x_{n}, z_{n}\right) \rightarrow(x, z)$ in 
$X \times Z$, then we have

$$
\begin{gathered}
d\left(z_{n}, F\left(t, x_{n}\right)\right)=d\left(z_{n}, F\left(t, x_{n}\right)\right)-d\left(z, F\left(t, x_{n}\right)\right)+d\left(z, F\left(t, x_{n}\right)\right) \\
\leq\left\|z_{n}-z\right\|+d\left(z, F\left(t, x_{n}\right)\right) \\
\Rightarrow \varlimsup \\
\lim d\left(z_{n}, F\left(t, x_{n}\right)\right) \leq \varlimsup \lim d\left(z, F\left(t, x_{n}\right)\right) \leq d(z, F(t, x)),
\end{gathered}
$$

the last inequality being a consequence of the hypothesis that $x \rightarrow F(t, x)$ is 1.s.c. (see for example Aubin-Cellina [1], Corollary 1, p. 52). So $(t, x, z) \rightarrow$ $d(z, F(t, x))$ is a measurable integrand which is u.s.c. in $(x, z)$. Then define

$$
\gamma_{k}(t, x, z)=\sup \left[\gamma(t, x, z)-k d_{X}\left(x, x^{\prime}\right)-k d_{Z}\left(z, z^{\prime}\right): x^{\prime} \in X, z^{\prime} \in Z\right]
$$

(here $d_{X}(\cdot, \cdot)$ denotes the metric on $X$ and $d_{Z}(\cdot, \cdot)$ the metric on $Z$ ).

From Lemma 2.1 of Hiai-Umegaki [7], we know that $t \rightarrow \gamma_{k}(t, x, z)$ is measurable, while it is easy to see that $(x, z) \rightarrow \gamma_{k}(t, x, z)$ is $k$-Lipschitz and $\gamma_{k} \downarrow \gamma$ as $k \rightarrow \infty$ (see for example Bertsekas-Shreve [2], Lemma 7.7, p. 125). Given $\varepsilon>0$, apply the classical Scorza-Dragoni theorem to find $T_{\varepsilon} \subseteq T$ compact with $\lambda\left(T_{\varepsilon}^{c}\right)<\varepsilon$ such that $\gamma_{k} \mid T_{\varepsilon} \times X \times Z$ is continuous for all $k \geq 1$. Since $\gamma_{k} \downarrow \gamma$, we get that $\left.\gamma\right|_{T_{\varepsilon} \times X \times Z}$ is u.s.c. (cf. Bertsekas-Shreve [2], Lemma 7.14, p. 147). So $\left.d(z, F(\cdot, \cdot))\right|_{T_{\varepsilon} \times X}$ is u.s.c. $\left.\Rightarrow F\right|_{T_{\varepsilon} \times X}$ is 1.s.c. (see Klein-Thompson [8]).

In the next result $\stackrel{\circ}{B}_{\varepsilon}(x)=\left\{y \in X: d_{X}(y, x)<\varepsilon\right\}$ (the open ball of radius $\varepsilon$, centered at $x \in X)$.

Proposition 3.2. If $X$ is a metric space, $h: X \rightarrow \mathbb{R}^{N}$ satisfies $\|h(x)\| \leq M$ for all $x \in X$ and we define $G(x)=\bigcap_{\varepsilon>0} \overline{\operatorname{conv}} h\left(\stackrel{\circ}{B}_{\varepsilon}(x)\right)$, then $x \rightarrow G(x)$ is u.s.c. from $X$ into $P_{k c}\left(\mathbb{R}^{N}\right)$.

Proof. Since $G(\cdot)$ is $P_{k c}\left(\mathbb{R}^{N}\right)$-valued, to establish its upper semicontinuity, it is enough to show that for every $x^{*} \in \mathbb{R}^{N}$,

$$
x \rightarrow \sigma\left(x^{*}, G(x)\right)=\sup \left\{\left\langle x^{*}, v\right\rangle_{\mathbb{R}^{N}}: v \in G(x)\right\}
$$

is u.s.c. (cf. Klein-Thompson [8]; here $\langle\cdot, \cdot\rangle_{\mathbb{R}^{N}}$ denotes the Euclidean inner product in $\left.\mathbb{R}^{N}\right)$. So fix $x^{*} \in \mathbb{R}^{N}$. We will show that for every $\lambda \in \mathbb{R}$, the level set $U_{\lambda}=\left\{x \in X: \lambda \leq \sigma\left(x^{*}, G(x)\right)\right\}$ is closed. To this end let $\left\{x_{n}\right\}_{n \geq 1} \subseteq U_{\lambda}$ and assume that $x_{n} \rightarrow x$ in $X$. Find $y_{n} \in G\left(x_{n}\right)$ such that $\lambda \leq\left\langle x^{*}, y_{n}\right\rangle_{\mathbb{R}^{N}}$. Let $\varepsilon_{n}=2 d_{X}\left(x_{n}, x\right) \downarrow 0$ as $n \rightarrow \infty$. Then we have $y_{n} \in G\left(\stackrel{\circ}{B}_{\varepsilon_{n}}(x)\right)$. Since $\left\|y_{n}\right\| \leq M, n \geq 1$, by passing to a subsequence if necessary we may assume that $y_{n} \rightarrow y$. Clearly then $y \in G(x)$ and $\left\langle x^{*}, y_{n}\right\rangle_{\mathbb{R}^{N}} \rightarrow\left\langle x^{*}, y_{n}\right\rangle_{\mathbb{R}^{N}} \Rightarrow \lambda \leq$ $\left\langle x^{*}, y\right\rangle_{\mathbb{R}^{N}}$ and so $\lambda \leq \sigma\left(x^{*}, G(x)\right)$. Therefore $\lambda \in U_{\lambda}$, which implies that $x \rightarrow \sigma\left(x^{*}, G(x)\right)$ is u.s.c. $\Rightarrow x \rightarrow G(x)$ is u.s.c.

For the next auxiliary result we will need the following hypothesis.

$$
H(K): K: T \rightarrow P_{k c}\left(\mathbb{R}^{N}\right) \text { is an } h \text {-Lipschitz multifunction. }
$$

Proposition 3.3. If hypothesis $H(K)$ holds, $G: T \times \mathbb{R}^{N} \rightarrow P_{k c}\left(\mathbb{R}^{N}\right)$ is a multifunction such that for all $(t, x) \in \mathrm{Gr} K, t<b, G(t, x) \subset D K(t, x)(1)$ and $x$ : $T \rightarrow \mathbb{R}^{N}$ is an absolutely continuous function such that $\dot{x}(t) \in G(t, p(t, x(t)))$ 
a.e., $x(0) \in K(0)$ (here $p(t, x)=\operatorname{proj}(x ; K(t))$, the metric projection on $K(t))$, then for all $t \in T, x(t) \in K(t)$.

Proof. Let $\varphi(t)=d(x(t), K(t))$. Using hypothesis $H(K)$, we can easily check that $\varphi(\cdot)$ is absolutely continuous on $T$, with $\varphi(0)=0$. So to prove our proposition, we only need to show that $\dot{\varphi}(t) \leq 0$ a.e.

To this end, let $t \in[0, b)$ be a point at which both $\dot{x}(\cdot)$ and $\dot{\varphi}(\cdot)$ exist. Then we have

$$
\begin{aligned}
\frac{\varphi(t+h)-\varphi(t)}{h} & =\frac{d(x(t+h), K(t+h))-d(x(t), K(t))}{h} \\
& =\frac{d(x(t)+h \dot{x}(t)+o(h), K(t+h))-d(x(t), K(t))}{h} \\
& \leq \frac{\|o(h)\|}{h}+\frac{d(x(t)+h \dot{x}(t), K(t+h))-d(x(t), K(t))}{h} .
\end{aligned}
$$

Observe that

$$
\begin{aligned}
& \frac{d(x(t)+h \dot{x}(t), K(t+h))-d(x(t), K(t))}{h} \\
& \leq \frac{1}{h}[\|x(t)-p(t, x(t))\|+d(p(t, x(t))+h \dot{x}(t), K(t+h))-d(x(t), K(t))] \\
& \quad=\frac{1}{h} d(p(t, x(t))+h \dot{x}(t), K(t+h)) \\
& \quad=d\left(\dot{x}(t), \frac{K(t+h)-p(t, x(t))}{h}\right) .
\end{aligned}
$$

Since by hypothesis $\dot{x}(t) \in G(t, p(t, x(t))) \subseteq D K(t, p(t, x(t)))(1)$ a.e. on $[0, b)$, from Aubin-Cellina [1], pp. 190-191, we have that

$$
\begin{aligned}
& \frac{\lim }{\lambda \downarrow 0} d\left(\dot{x}(t), \frac{K(t+h)-p}{h} \frac{(t, x(t))}{2}\right)=0 \text { a.e. } \\
& \Rightarrow \dot{\varphi}(t) \leq 0 \text { a.e. } \\
& \Rightarrow \varphi(t)=0 \text { for all } t \in T \text { and so } x(t) \in K(t) \text { for all } t \in T
\end{aligned}
$$

The next auxiliary result establishes a useful property of lower semicontinuous multifunctions and will lead us to a new lower semicontinuity result concerning the intersection of two multifunctions.

Proposition 3.4. If $Z$ is a Hausdorff topological space, $F: Z \rightarrow 2^{\mathbb{R}^{N}} \backslash\{\varnothing\}$ is l.s.c. with convex values and $\stackrel{\circ}{B}(x, \hat{r})=\left\{y \in \mathbb{R}^{N}:\|y-x\|<\hat{r}\right\} \subseteq F\left(z_{0}\right)$, then for all $r \in(0, \hat{r})$, there exists an open neighborhood $U$ of $z_{0}$ such that $\stackrel{\circ}{B}(x, r) \subseteq F(z)$ for all $z \in U$.

Proof. Let $r \in(0, \hat{r})$ and take $r^{\prime}, \varepsilon>0$ such that $0<r<r^{\prime}<\hat{r}$ and $0<\varepsilon<r^{\prime}-r$. We have $\stackrel{\circ}{B}\left(x, r^{\prime}\right) \subseteq F\left(z_{0}\right)$. Let $\theta(z)=h^{*}\left(B\left(x, r^{\prime}\right), F(z)\right)=$ $\sup \left[d(v, F(z)): v \in B\left(x, r^{\prime}\right)\right]$. We know (cf. Aubin-Cellina [1], Theorem 5, p. 52) that $\theta(\cdot)$ is u.s.c. and $\theta\left(z_{0}\right)=0$. So we can find $U$ an open neighborhood of $z_{0}$ such that $\theta(z)<\varepsilon$ for all $z \in U$. Since by hypothesis $F(\cdot)$ is convex- 
valued from Moreau [11], we know that

$$
\begin{aligned}
& d\left(x, \mathbb{R}^{N} \backslash \stackrel{\circ}{B}\left(x, r^{\prime}\right)\right)-d\left(x, \mathbb{R}^{N} \backslash F(z)\right) \leq h^{*}\left(B\left(x, r^{\prime}\right), F(z)\right) \\
& =\theta(z)<\varepsilon \text { for all } z \in U, \\
& \Rightarrow d\left(x, \mathbb{R}^{N} \backslash \stackrel{\circ}{B}\left(x, r^{\prime}\right)\right)-\varepsilon \leq d\left(x, \mathbb{R}^{N} \backslash F(z)\right), \\
& \Rightarrow r<r^{\prime}-\varepsilon \leq d\left(x, \mathbb{R}^{N} \backslash F(z)\right) \text { for all } z \in U \text {. }
\end{aligned}
$$

So we conclude that for all $z \in U, \stackrel{\circ}{B}(x, r) \subseteq F(z)$.

Having this proposition, we can now prove a lower semicontinuity result for the intersection of two multifunctions.

Proposition 3.5. If $Z$ is a Hausdorff topological space, $H_{1}, H_{2}: Z \rightarrow 2^{\mathbb{R}^{N}} \backslash\{\varnothing\}$ are l.s.c. multifunctions such that for all $z \in Z, H_{2}(z)$ is open and convex and $H_{1}(z) \cap H_{2}(z) \neq \varnothing$, then $z \rightarrow H(z)=H_{1}(z) \cap H_{2}(z)$ is l.s.c.

Proof. We claim that $\mathrm{Gr} \mathrm{H}_{2}$ is open. Indeed let $(z, v) \in \mathrm{Gr} \mathrm{H}_{2}$. Then $v \in$ $H_{2}(z)$ and so we can find $\hat{r}>0$ such that $\stackrel{\circ}{B}(v, \hat{r}) \subseteq H_{2}(z)$. Let $r<\hat{r}$ and apply Proposition 3.4 to get $U$ an open neighborhood of $z$ such that $\stackrel{\circ}{B}(v, r) \subseteq H_{2}\left(z^{\prime}\right)$ for all $z^{\prime} \in U$. Set $W=U \times \stackrel{\circ}{B}(v, r)$. Clearly this is an open neighborhood of $(z, v) \in Z \times \mathbb{R}^{N}$ and for every $\left(z^{\prime}, v^{\prime}\right) \in W$ we have $v^{\prime} \in$ $\stackrel{\circ}{B}(v, r) \subseteq H_{2}\left(z^{\prime}\right)$. So $\left(z^{\prime}, v^{\prime}\right) \in \operatorname{Gr} H_{2}$ for all $\left(z^{\prime}, v^{\prime}\right) \in W \Rightarrow \operatorname{Gr} H_{2}$ is open and so by the lemma in Papageorgiou [13], we conclude that $z \rightarrow H_{1}(z) \cap H_{2}(z)$ must be l.s.c.

Let $\Gamma$ be a cone in $\mathbb{R}^{m}$ and $X$ a metric space. Following Bressan [3], we say that the single-valued map $f: \mathbb{R}^{m} \rightarrow X$ is $\Gamma$-continuous at $v \in \mathbb{R}^{m}$, if and only if $f\left(v_{n}\right) \rightarrow f(v)$ when $v_{n} \rightarrow v$ in $\mathbb{R}^{m}$ and $v_{n}-v \in \Gamma$ for all $n \geq 1$ (equivalently for every $\varepsilon>0$, there exists $\delta>0$ such that $d_{X}\left(f(v), f\left(v^{\prime}\right)\right)<\varepsilon$ when $\left.\left\|v-v^{\prime}\right\|<\delta, v^{\prime}-v \in \Gamma\right)$. We will say that $f(\cdot)$ is $\Gamma$-continuous, if it is $\Gamma$-continuous at every $v \in \mathbb{R}^{m}$. Let $M>0$ and on $\mathbb{R} \times \mathbb{R}^{N}$ consider the cone $\Gamma^{M}=\left\{(t, x) \in \mathbb{R} \times \mathbb{R}^{N}:\|x\| \leq M t\right\}$. A map $h: T \times \mathbb{R}^{N} \rightarrow \mathbb{R}^{N}$ is said to be Scorza-Dragoni $\Gamma^{M}$-continuous, if for every $\varepsilon>0$, we can find $T_{\varepsilon} \subseteq T$ compact such that $\lambda\left(T_{\varepsilon}^{c}\right)<\varepsilon$ and $\left.h\right|_{T_{\varepsilon} \times \mathbb{R}^{N}}$ is $\Gamma^{M}$-continuous.

Now let $h: T \times \mathbb{R}^{N} \rightarrow \mathbb{R}^{N}$ be a Scorza-Dragoni $\Gamma^{M}$-continuous map such that $\|h(t, x)\| \leq M$ for all $(t, x) \in T \times \mathbb{R}^{N}$. Let $G(t, x)=\bigcap_{\varepsilon>0} \overline{\operatorname{conv}} h\left(\stackrel{\circ}{B}_{\varepsilon}(t, x)\right)$ (cf. Proposition 3.2). We consider the following two Cauchy problems:

$$
\left\{\begin{array}{c}
\dot{x}(t) \in G(t, x(t)) \text { a.e. } \\
x(0)=x_{0}
\end{array}\right\}
$$

and

$$
\left\{\begin{array}{c}
\dot{x}(t)=h(t, x(t)) \text { a.e. } \\
x(0)=x_{0} .
\end{array}\right\}
$$

We have the following result relating these two problems. 
Proposition 3.6. If $x: T \rightarrow \mathbb{R}^{N}$ is a solution of (2), then $x(\cdot)$ also solves (3). Proof. From Lusin's theorem and the fact that $h(\cdot, \cdot)$ is Scorza-Dragoni $\Gamma^{M}$. continuous, we can find $T_{n} \subseteq T, n \geq 1$, measurable sets such that $\left.\dot{x}\right|_{T_{n}}$ is continuous, $\left.h\right|_{T_{n} \times \mathbb{R}^{N}}$ is $\Gamma^{M}$-continuous, $\dot{x}(t) \in G(t, x(t))$ for all $t \in T_{n}$ and $\lambda\left(T \backslash \bigcup_{n \geq 1} T_{n}\right)=0$. Also invoking Lebesgue's density theorem (see Oxtoby [12]), we can find sets $N_{n} \subseteq T_{n}$, with $\lambda\left(N_{n}\right)=0$, such that every $t \in T_{n} \backslash N_{n}$ is a density point for $T_{n}$. Next let $t \in T_{n} \backslash N_{n}$. We can find $t_{m} \in T_{n} \backslash N_{n}$, $t_{m}>t, m \geq 1$, and $t_{m} \downarrow t$ as $m \rightarrow \infty$. So $\dot{x}\left(t_{m}\right) \rightarrow \dot{x}(t)$. Also because by hypothesis $\|h(t, x)\| \leq M$, we have $|G(t, x)|=\sup \{\|v\|: v \in G(t, x)\} \leq M$ and so $\|x(t)-x(s)\| \leq M|t-s|$ for all $t, s \in T$. Let $\varepsilon>0$. We have $\dot{x}\left(t_{m}\right) \in$ $G\left(t_{m}, x\left(t_{m}\right)\right) \subseteq h\left(t_{m}, x\left(t_{m}\right)\right)+(\varepsilon / 2) \stackrel{\circ}{B}_{1}$, where $\stackrel{\circ}{B}_{1}=\left\{y \in \mathbb{R}^{N}:\|y\|<1\right\}$. Since $\left.h\right|_{T_{n} \times \mathbb{R}^{N}}$ is $\Gamma^{\bar{M}}$-continuous, we can find $m_{0}(\varepsilon) \geq 1$ such that for $m \geq m_{0}$ we have

$$
\begin{aligned}
& \left\|h\left(t_{m}, x\left(t_{m}\right)\right)-h(t, x(t))\right\|<\frac{\varepsilon}{2} \\
& \quad \Rightarrow h\left(t_{m}, x\left(t_{m}\right)\right) \in h(t, x(t))+\frac{\varepsilon}{2} \stackrel{\circ}{B}_{1} .
\end{aligned}
$$

Hence for $m \geq m_{0}(\varepsilon)$ we have

$$
\begin{aligned}
\dot{x}\left(t_{m}\right) & \in h(t, x(t))+\varepsilon \stackrel{\circ}{B}_{1} \\
\Rightarrow & \dot{x}(t) \in h(t, x(t))+\varepsilon B_{1} .
\end{aligned}
$$

Let $\varepsilon \downarrow 0$, to get that for all $t \in \widehat{T}=\bigcup_{n \geq 1}\left(T_{n} \backslash N_{n}\right), \lambda(T \backslash \widehat{T})=0$, we have $\dot{x}(t)=h(t, x(t)), x(0)=x_{0} \Rightarrow x(\cdot)$ is a solution of $(3)$.

\section{MAIN THEOREM}

In this section we state and prove our main result, concerning the existence of solutions for problem (1). For this we will need the following hypotheses: $H(F): F: T \times \mathbb{R}^{N} \rightarrow P_{k}\left(\mathbb{R}^{N}\right)$ is a multifunction such that

(1) $(t, x) \rightarrow F(t, x)$ is measurable,

(2) $x \rightarrow F(t, x)$ is l.s.c.,

(3) $|F(t, x)|=\sup \{\|v\|: v \in F(t, x)\} \leq M$ for all $(t, x) \in T \times \mathbb{R}^{N}$.

$H(K)^{\prime}: K: T \rightarrow P_{k c}\left(\mathbb{R}^{N}\right)$ is an $h$-Lipschitz multifunction such that $K(0) \supseteq$ $K(b),(t, x) \rightarrow D K(t, x)(1)$ is 1.s.c. on $\operatorname{Gr} K \cap\left([0, b) \times \mathbb{R}^{N}\right)$ and for every $(t, x) \in \mathrm{Gr} K \cap\left([0, b) \times \mathbb{R}^{N}\right)$ we have int $D K(t, x)(1) \neq \varnothing$.

Remark. Note that if $K(t)=K \in P_{k c}\left(\mathbb{R}^{N}\right), t \in T$, and int $K \neq \varnothing$, then hypothesis $H(K)^{\prime}$ is automatically satisfied. To see this, remark that $D K(t, x)(1)$ $=\operatorname{proj}_{\mathbb{R}^{N}}\left(\left[\{1\} \times \mathbb{R}^{N}\right] \cap T_{\mathrm{Gr} K}(t, x)\right)=\operatorname{proj}_{\mathbb{R}^{N}}\left(\left[\{1\} \times \mathbb{R}^{N}\right] \cap\left[T_{T}(x) \times T_{K}(x)\right]\right)=T_{K}(x)$ and apply Theorem 1 and Proposition 4, pp. 220-221, of Aubin-Cellina [1]. Also note that our lower semicontinuity hypothesis on $(t, x) \rightarrow D K(t, x)(1)$ implies that this multifunction is convex-valued (cf. Aubin-Cellina [1]).

$H_{\tau}:$ For all $(t, x) \in \operatorname{Gr} K, t<b$, we have $F(t, x) \cap$ int $D K(t, x)(1) \neq \varnothing$ (tangential condition).

Theorem 4.1. If hypotheses $H(F), H(K)^{\prime}$ and $H_{\tau}$ hold, then problem (1) admits a solution. 
Proof. From Proposition 3.5, we have that $x \rightarrow F(t, x) \cap$ int $D K(t, x)(1)$ is 1.s.c. $\Rightarrow x \rightarrow \overline{F(t, x) \cap \text { int } D K(t, x)(1)}$ is 1.s.c. Also because of hypothesis $H(F)(1)$, we have that $(t, x) \rightarrow \overline{F(t, x) \cap \operatorname{int} D K(t, x)(1)}$ is measurable. Let $H(t, x)=\overline{F(t, x) \cap \text { int } D K(t, x)(1)}$. From Proposition 3.1 we know that given $\varepsilon>0$, we can find $T_{\varepsilon} \subseteq T$ compact with $\lambda\left(T_{\varepsilon}^{c}\right)<\varepsilon$ such that $\left.H\right|_{T_{\varepsilon} \times \mathbb{R}^{N}}$ is 1.s.c. So we can apply Theorem 1 of Bressan [3] and get a function $h: T \times \mathbb{R}^{N} \rightarrow \mathbb{R}^{N}$ such that $h(\cdot, \cdot)$ is Scorza-Dragoni $\Gamma^{M}$-continuous and for all $(t, x) \in T \times$ $\mathbb{R}^{N}, h(t, x) \in H(t, x)$ (recall $\Gamma^{M}$ is the cone $\left\{(t, x) \in \mathbb{R}_{+} \times \mathbb{R}^{N}:\|x\| \leq M t\right\}$ ). Then consider the multifunction $G(t, x)=\bigcap_{\varepsilon>0} \overline{\operatorname{conv}} h\left(\stackrel{\circ}{B}_{\varepsilon}(t, x)\right)$, where $\stackrel{\circ}{B}_{\varepsilon}(t, x)=\left\{\left(t^{\prime}, x^{\prime}\right) \in T \times \mathbb{R}^{N}:\left|t-t^{\prime}\right|<\varepsilon,\left\|x-x^{\prime}\right\|<\varepsilon\right\}$. From Proposition 3.2 we know that $(t, x) \rightarrow G(t, x)$ is u.s.c., and clearly from the above definition we have that $G(t, x) \subseteq \overline{\operatorname{conv}} F(t, x) \subseteq D K(t, x)(1)$ for all $(t, x) \in \operatorname{Gr} K$, $t<b$. Then consider the following Cauchy problem:

$$
\left\{\begin{array}{c}
\dot{x}(t) \in G(t, p(t, x(t))) \text { a.e. } \\
x(0)=z \in K(0) .
\end{array}\right\}
$$

It is well known (see for example, DeBlasi-Myjak [4] and Gorniewicz [5]) that the solution set $S(z)$ of $(4)$ is an $R_{\delta}$-set in $C\left(T, \mathbb{R}^{N}\right)$. In particular, then it is compact and acyclic (with respect to the Čech cohomology; cf. Proposition 3.1 of Gorniewicz [5]). Furthermore from Proposition 3.3, we know that $S(z)(b)=$ $\{x(b): x \in S(z)\} \subseteq K(b) \subseteq K(0)$. Let $e_{b}: C\left(T, \mathbb{R}^{N}\right) \rightarrow \mathbb{R}^{N}$ be the evaluation at $b$ map; i.e. $e_{b}(x)=x(b)$. Let $R=e_{b} \circ S: K(0) \rightarrow P_{k}(K(0))$. This is a pseudo-acyclic map in the sense of Lasry-Robert [9] (see Definition 5), and so by Theorem 7 of that paper we can find $z \in R(z)$. Let $x \in S(z)$ such that $x(0)=x(b)=z$. Then $\dot{x}(t) \in G(t, p(t, x(t)))$ a.e., $x(0)=x(b)$. Recalling that $G(t, x) \subseteq D K(t, x)(1)$ for $(t, x) \in \mathrm{Gr} K, t<b$, from Proposition 3.3 we get that $x(t) \in K(t)$ for all $t \in T$. So $\dot{x}(t) \in G(t, x(t))$ a.e., $x(0)=x(b)$. Invoking Proposition 3.6, we get $\dot{x}(t)=h(t, x(t)) \in F(t, x(t))$ a.e., $x(0)=$ $x(b)$; i.e. $x(\cdot)$ solves $(1)$.

\section{ACKNOWLEDGMENT}

The authors wish to thank the referee for his (her) corrections.

\section{REFERENCES}

1. J.-P. Aubin and A. Cellina, Differential inclusions, Springer-Verlag, Berlin, 1984.

2. D. Bertsekas and S. Shreve, Stochastic optimal control: The discrete time case, Academic Press, New York, 1978.

3. A. Bressan, Directionally continuous selections and differential inclusions, Funkcial. Ekvac. 31 (1988), 459-470.

4. F. S. DeBlasi and J. Myjak, On the solution sets for differential inclusions, Bull. Polish Acad. Sci. 33 (1985), 17-23.

5. L. Gorniewicz, On the solution sets of differential inclusions, J. Math. Anal. Appl. 113 (1986), 235-244.

6. G. Haddad and J.-M. Lasry, Periodic solutions of functional-differential inclusions and fixed points of $\sigma$-selectionable correspondences, J. Math. Anal. Appl. 96 (1983), 295-312.

7. F. Hiai and H. Umegaki, Integrals, conditional expectations and martingales of multivalued functions, J. Multivariate Anal. 7 (1977), 149-182. 
8. E. Klein and A. Thompson, Theory of correspondences, Wiley-Interscience, New York, 1984.

9. J.-M. Lasry and R. Robert, Degré topologique pour certains couples de fonctions et applications aux equations differentielles multivoques, C. R. Acad. Sci. Paris 283 (1976), 163-166.

10. J. Macki, P. Nistri, and P. Zecca, The existence of periodic solutions to nonautonomous differential inclusions, Proc. Amer. Math. Soc. 104 (1988), 840-844.

11. J.-J. Moreau, Intersection of moving convex sets in a normed space, Math. Scand. 36 (1975), 159-173.

12. J. Oxtoby, Measure and category, Springer-Verlag, New York, 1971.

13. N. S. Papageorgiou, On the existence of $\psi$-maximal, viable solutions for a class of differential inclusions, Arch. Math. (Brno) 27 (1991), 175-182.

14. S. Plaskacz, Periodic solutions of differential inclusions on compact subsets of $\mathbb{R}^{n}$, J. Math. Anal. Appl. 148 (1990), 202-212.

Department of Mathematics, Southwest Missouri State University, Springfield, Missouri 65804

E-mail address: $\operatorname{shh} 209 \mathrm{f} \odot \mathrm{cnas} . \mathrm{smsu} . \mathrm{edu}$

Department of Mathematics, National Technical University, Zografou Campus, AthENS 15773, GREECE 